\title{
MISCELÁNEAS
}

\section{El concepto de riesgo sexual al interior de las Ciencias Sociales: una clave interpretativa para re-pensar las políticas de prevención en VIH/SIDA}

\section{Claudia Moreno Standen ${ }^{1}$}

El SIDA no es una enfermedad cualquiera. De hecho, no es solo una enfermedad en particular sino que un síndrome de ellas. Su especificidad no reside exclusivamente en su carácter epidémico, al cual la tecnología biomédica no ha podido encontrar una cura, y que, hasta la aparición de las terapias antiretrovirales, llevaba a quienes la padecían a una muerte inexorable. Como señala Jeffrey Weeks (2000) esta es una enfermedad que a pesar de su reciente aparición, lleva consigo una alta carga de historias. Primero, por su connotación altamente sexual, debido a que es una de las formas más efectivas de transmisión de la enfermedad, y a que los primeros casos se presentaron en grupos de hombres homosexuales, una comunidad altamente sexualizada, el SIDA emergió en un momento particular de la llamada historia de la sexualidad, de importantes flujos y cambios en los valores sexuales y en los patrones de interacción sexual. En segundo término, por los desafíos que la epidemia ha planteado no solo a la investigación biológica y farmacológica, sino que a la investigación social; la crisis que ha generado sobre las políticas económicas, sociales y de bienestar, etc. Su carácter queda bien descrito en las palabras de Jonathan Mann, "la epidemia del SIDA se ha extendido por todo el mundo siguiendo siempre las fisuras de cada sociedad." En todo sentido, el SIDA sobrepasa lo que podría ser una simple emergencia epidemiológica, complejizando y desafiando todos los desarrollos éticos, pedagógicos y sociales de la prevención, tal como ha sido entendida en la salud pública y en las ciencias sociales. 
La prioridad de la prevención en el caso del VIH/SIDA se vincula a la inexistencia de una vacuna que inmunice a las personas del virus, o bien del desarrollo de algún medicamento que cure en forma definitiva la enfermedad. A pesar del espectacular avance de la industria farmacológica en relación a las terapias antiretrovirales, que hasta el momento han demostrado ser capaces de mantener la enfermedad en un estado crónico, no existen indicios de que alguna de las opciones anteriores -vacuna o cura- vayan a ser descubiertas próximamente. Por otra parte, la dificultad de acceso a los fármacos existentes, debido a sus altos costos, exige concentrar gran parte de los esfuerzos en las intervenciones preventivas. El problema de una respuesta efectiva al VIH/SIDA de ningún modo queda reducido a una cuestión técnica, quedando demostrado desde el inicio de la década de los noventa la importancia de la investigación social y de la producción de conocimiento para la comprensión de la epidemia, no solo en el ámbito de la prevención sino en temas más generales en lo relativo a las formas de organización, tanto materiales como simbólicas, de la sexualidad, la construcción de las identidades sexuales, la constitución de comunidades sexuales, la vinculación de la sexualidad a los sistemas sexo-genéricos, etc.

Cuando hace ya veinte años comenzaron a presentarse los primeros casos de VIH/SIDA en el mundo, nadie imaginó la rapidez de su avance. El hecho de que los primeros casos de personas viviendo con el VIH/SIDA se presentaran en varones homosexuales, usuarios de drogas intravenosas, pobres urbanos, y otros, hizo pensar que el virus solo se manifestaba en ciertos contextos poblacionales más bien marginales, lo que retrasó una política de prevención orientada hacia la población en general. Esto propició además la instalación de la idea de lo sexual como causa de la enfermedad y no como vía de transmisión de la misma, ayudando a la estigmatización de la epidemia. Una vez en marcha, las políticas y estrategias de prevención para el VIH/SIDA han tenido un desarrollo acelerado, en gran medida porque la lógica de la epidemia ha llevado a un continuo cuestionamiento de las herramientas hasta este momento utilizadas.

Sin embargo, la discusión no puede quedar remitida al ámbito técnico de las estrategias, de los diseños de intervención pedagógicos. La reflexión debe aproximarse al campo de lo 
epistemológico, con el objeto de pensar cómo estas intervenciones, ancladas a un referente teórico particular, suponen, la mayor parte de las veces de manera implícita, una determinada concepción del riesgo y del sujeto. El desafío que nos propone un ejercicio de develamiento de las concepciones de riesgo y sujeto implícitas en estas teorías y estas prácticas, tiene una finalidad ética y práctica; toda vez que reproducen ciertas normas sociales, protegen instituciones, producen y reproducen determinadas relaciones de poder.

El objetivo de este artículo es hacer el ejercicio teórico de revisar los enfoques en prevención que se han utilizado para el $\mathrm{VIH} / \mathrm{SIDA}$, con el fin de hacer explícitos las nociones de riesgo sexual y de subjetividad que en ellos se juegan. En la medida que sea posible develar dicha articulación, será posible reconocer los alcances de tales enfoques, ya sea desde su crítica o desde sus aportes. Antes de aproximarnos a los distintos enfoques de prevención, nos centraremos en algunos aspectos generales del concepto de riesgo desde la salud y desde las ciencias sociales. Esto en el entendido de que la categoría de riesgo sexual remite directamente a la noción de riesgo en salud, utilizada en la epidemiología y la salud pública; por otra parte, utilizaremos las referencias de la teoría social en torno al tema, lo que nos permitirá tener un marco de referencia mayor a la hora de revisar su instrumentalización en los abordajes teóricos de la prevención en salud. Luego, intentaremos dar cuenta de aquellos enfoques que han sido más recurrentes en las políticas y estrategias de prevención, intentando trazar una cierta historicidad conceptual entre ellos, que nos permita visibilizar los conceptos de riesgo y sujeto en ellas contenidos, de modo que sirva para una reflexión en torno a las consecuencias de la epidemia del VIH/SIDA.

\section{Una aproximación al concepto de riesgo}

El riesgo en salud. El riesgo es un concepto central para la epidemiología y la salud pública, siendo utilizado para describir los patrones diferenciales de morbilidad o mortalidad asociados con un evento. Técnicamente, el término riesgo se refiere al exceso 
de morbilidad o mortalidad asociado con la exposición a un ambiente, condición o patógeno. De una manera más amplia, es definido como un "fenómeno interno, maleable y manejable por la misma persona, manifestado por presencia o ausencia de control individual frente a la exposición del organismo a determinadas contingencias que conllevan un riesgo de contagio" (Conasida 2000, 217). De esta perspectiva, el riesgo no solo lo constituye la exposición al patógeno en sí, sino que la falta de habilidades de control en la propia conducta. Como señala Kendall (1995), a estos análisis expertos sobre el riesgo subyace el modelo dicotómico de sano y enfermo, normal y anormal, cuerpo y ambiente, etc.

Sucede que a pesar de su centralidad, o quizás debido a ella, el concepto de riesgo es escasamente explorado en relación a sus significaciones e implicancias. En general, en los estudios que exploran teorías y / o intervenciones en la línea de la prevención del VIH/SIDA, rara vez se explicita el uso del término riesgo, el que sin embargo, sí es usado con frecuencia. Tampoco se hace referencia a la investigaciones de la teoría social que han estudiado el tema del riesgo, aun cuando las referencias a los desarrollos de las ciencias sociales sobre los conceptos de salud y enfermedad, la centralidad del discurso biomédico en las sociedad modernas, o el énfasis de la sexualidad como una construcción social y simbólica han sido ampliamente incorporados.

\section{El riesgo desde la teoría social}

Diversos autores sostienen que la construcción del riesgo como concepto, es un evento reciente, una manifestación del modernismo, que reedita aquellos conceptos tradicionales de la suerte, fortuna y destino (Giddens, 1996). El concepto de riesgo como tal se liga a la concepción moderna de racionalidad y a la concepción temporal de futuro. En este contexto, el riesgo tiene la pretensión de convertirse en una unidad de medida del enfrentamiento del peligro y la búsqueda de la seguridad social. Con el avance de la modernidad, y el advenimiento de la modernidad tardía, éste se masifica en su uso y en la experiencia cotidiana, alcanzando los más diversos órdenes sociales (trabajo, salud, re- 
laciones de pareja, sexo, etc.). Dentro del marco referencial de la modernidad, adquiere importancia el proceso de industrialización a partir del siglo XVIII. A este respecto, Ulrich Beck (1996) plantea que desde ese hito habría comenzado a germinar lo que él denomina una sociedad del riesgo. Este concepto hace referencia a una doble experiencia en la sociedad industrializada actual: primero, por la posibilidad cada día mayor de que se produzcan daños que afecten a buena parte de la humanidad, asociados a la universalización de la tecnología y a la globalización. De esta forma, nos encaminaríamos hacia una nueva modernidad en la que el eje que estructura nuestra sociedad industrial ya no es la clásica distribución de bienes, "sino la distribución de males, nos guste o no, es la distribución del riesgo más que de la riqueza lo que moviliza hoy a numerosos colectivos sociales" (López y Lujan 2000, 20). En segundo término, la sociedad del riesgo, consiste en la presencia cada vez mayor de decisiones arriesgadas dentro de la conducta cotidiana. Para Beck (1998), "la sociedad del riesgo comienza donde termina la tradición, cuando, en todas las esferas de la vida ya no podemos dar por supuestas las certidumbres tradicionales. Cuando menos podemos confiar en las seguridades tradicionales, más riesgos debemos negociar" (Cit. a Beck, 1998, en López y Lujan 2000, 21). Esta conceptualización nos ayuda a comprender por una parte la instrumentalización del riesgo; cómo éste se ha convertido en un eje central en el desarrollo de la salud pública, por ejemplo, y a la vez ilumina el camino para poder pensar en sus consecuencias políticas, al sostener la idea de la distribución de la responsabilidad y la culpa en nuestra sociedad.

Otra aproximación al concepto de riesgo desde las ciencias sociales la constituye su conceptualización como un constructo social e histórico. Dicho abordaje, desarrollado por Mary Douglas, nos parece central para comprender la importancia de este concepto en el diseño y desarrollo de estrategias de prevención para el VIH/SIDA, por la centralidad que éste tiene nos detendremos en su análisis posteriormente, con la finalidad de dialogar con los enfoques de prevención y de riesgo tradicionales. 


\section{La articulación del concepto de riesgo y las teorías de la prevención}

Si bien no es posible trazar un eje lineal en relación a la historia de los desarrollos teóricos sobre el riesgo y la prevención, conceptualmente se pueden delimitar distintos enfoques en la investigación social del VIH/SIDA. En este sentido, los enfoques que revisaremos no deben ser entendidos como una serie de fases sucesivas sino más bien como representantes de una amplitud de las investigaciones desarrolladas en relación al tema y, como señala Parker (2000), como indicativas de los tipos de problemas que deben ser considerados en el desarrollo de las nuevas agendas de investigación en VIH/SIDA.

1. Un primer enfoque para la prevención del VIH/SIDA fue el de la "racionalidad como comportamiento saludable" (Mere, 1999). La referencia principal de estas aproximaciones es el Modelo de Creencias en Salud (Health Belief Model). Este modelo tradicional de la salud pública, que incorpora los desarrollos de la psicología del comportamiento, fue inicialmente diseñado para una campaña finlandesa contra enfermedades cardiovasculares en los años cincuenta. Sostiene como tesis central que las acciones que los individuos emprenden en relación a su salud, están basadas en las creencias que manejan con respecto a ella. Por lo tanto, las intervenciones en esta perspectiva buscan influenciar el conocimiento, las actitudes y prácticas de los individuos a través de la entrega de la información adecuada, que muestre los costos y beneficios de determinados comportamientos para su salud. Si la persona valora su salud, cambiará su comportamiento. El objetivo es reducir el riesgo de exposición individual a la enfermedad.

En esta lógica, las personas serían individuos aislados que se comportan de forma natural como "ingenieros," midiendo y calculando los riesgos que se les presentan y tomando decisiones racionales frente a ellos. De ello, a su vez, se desprende que la sola información compensaría la debilidad cognitiva de las personas y su comprensión tendría como consecuencia la adopción de una conducta "saludable." Gran parte de las campañas de información masiva para el VIH/SIDA, tienen a la base esta lógica, de entrega de información. No solo se supone la racionalidad del 
individuo, sino que se lo define como autónomo, seguro de sí, gestor responsable de su tiempo, relaciones y necesidades. Por otra parte, reproduce la distinción entre cultura popular y saber científico, entre no-saber y saber.

2. Un segundo enfoque, que llamaremos enfoque de "la percepción de riesgo," viene a complejizar la mirada puramente racional e incorpora otros elementos subjetivos, así como elementos sociales a la prevención.

Si bien se reconoce la realidad objetiva del riesgo en los mismos términos que lo hace el enfoque racional, el riesgo es aquí entendido en términos de representación cognitiva; es decir, como un estado mental de agentes individuales (riesgo percibido), con probabilidades subjetivas y grados de aceptabilidad que dependen de una serie de variables contextuales vinculados a estados de creencias o disposiciones comportamentales del individuo.

Esta línea de investigación se ha ocupado particularmente de estudiar las variables que influyen individualmente sobre la percepción y valoración del riesgo, tratando de entender la forma en que las personas evalúan el riesgo en situaciones particulares. Esto es lo que se conoce también como 'riesgo subjetivo,' un tipo de riesgo que, de acuerdo con este enfoque, permite la toma de decisiones irracionales de aceptabilidad, es decir, que considera que las personas pueden comportarse arriesgadamente porque el cálculo costo-beneficio que operan sobre el riesgo, no es el mismo que ejercen los expertos.

La percepción individual del riesgo se erige desde este enfoque como la variable central de toda la conducta preventiva y hasta de toda la compleja gama de comportamientos relacionados con la salud ${ }^{1}$. Las intervenciones comportamentales bajo este paradigma se orientaron a modificar en los individuos la situación o magnitud de los principales 'determinantes' de las conductas sexuales, con la intención de producir un cambio en estas últimas. Diseñadas y puestas en prácticas estas intervenciones se orientaban primariamente a la entrega de información, a la motivación de la conciencia de riesgo y el deseo de reducirlo, y al desarrollo de habilidades para prácticas seguras, negociación sexual, etc.

Lo relevante de estas aproximaciones es que cuestionan las hipótesis cognitivas del ensayo-error y/o las limitaciones en los 
instrumentos utilizados en el procesamiento de la información y en el cálculo de probabilidades subjetivas como pieza clave y única del complejo proceso del riesgo. Sin embargo, una vez que el enfoque de la percepción de riesgo generó nuevas directrices para el abordaje de los riesgos, su aplicabilidad en el terreno del riesgo sexual no consiguió los objetivos planteados para la prevención. Especial importancia cobraron, en el cuestionamiento de este enfoque, los resultados negativos de la utilización del concepto de grupos de riesgo en los programas de prevención. La idea de que existirían sujetos que por sus estilos de vida, comportamientos y personalidad estarían más propensas a la adquisición del VIH, facilitó la marginación de poblaciones que, siendo vulnerables a la adquisición del $\mathrm{VIH}$, no fueron consideradas 'grupos de riesgo,' y por tanto fueron excluidas de los programas de prevención, y tuvo como consecuencia un aumento significativo de los casos de VIH/SIDA en mujeres, niños, hombres heterosexuales y jóvenes. Fracaso relativo, pues muchas intervenciones diseñadas desde esta perspectiva tuvieron resultados positivos en comunidades específicas, sin embargo, su debilidad quedaba demostrada a la hora intentar generalizar su utilización en campañas más masivas.

A pesar que estos modelos fueron más allá de la consideración del conocimiento como base para el cambio de comportamiento, y señalaban la existencia de otros factores psicológicos (emociones, motivaciones, actitudes, etc.), así como de elementos interaccionales tales como las presiones sociales, su unidad de análisis era el individuo, un individuo en un espacio social homogéneo, que no reconoce conflictos ni relaciones de poder (Cáceres, 1999). Entre ellas es posible reconocer la Teoría Social Cognitiva, el Modelo de las Creencias en Salud, Modelo de las Influencias Sociales, Teoría de la Acción Planificada (Ajzen y Madden), Autoeficacia Percibida (Bandura), Teoría de la Utilidad Esperada (Bajos).

3. Por último, es posible delimitar un tercer enfoque, comprendido por los abordajes más recientes en materia de prevención, que desarrollaremos siguiendo a Parker (2000). Al interior de esta propuesta se encuentran las Intervenciones Estructurales, que tienen como objetivo alterar las condiciones estructurales que impiden o facilitan la adopción de cambios en el comportamiento, por 
ejemplo ofrecer alternativas de trabajo a las personas que trabajan con sexo, o mejorar la logística de distribución de preservativos. Son estas intervenciones que sirven de base a otras estrategias.

También se ha dado paso a la Movilización Comunitaria, es decir, al estímulo del activismo por parte de las poblaciones, los que se abordan con estudios sobre la dinámica de la organización comunitaria. Su mérito es el de reconceptualizar los cambios en el comportamiento ocurridos a nivel comunitario no solo como resultado de la sumatoria del cambio de comportamientos individuales dentro de la comunidad, sino como una fuerza intersubjetiva e independiente que da forma a los cambios que suceden en las vidas individuales de las personas.

Por último, y de manera más innovadora se encuentran las Teorías de la Transformación social y empoderamiento colectivo, que analizan las cuestiones referentes al poder y la opresión. En este sentido, ha adquirido gran importancia el abordaje de la Educación Popular de Paulo Freiré, que postula el carácter transformador de los procesos de aprendizaje, a través del cual los participantes exploran y cuestionan sus propias vidas y realidades, experimentando así un proceso de capacitación y transformación colectiva a fin de reconocer las fuerzas que los amenazan y los oprimen.

Este último enfoque ha sido conceptualizado de una manera diferente a los llamados abordajes de la racionalidad y de la percepción del riesgo. En primer lugar, porque su emergencia se debe a las lecciones aprendidas dentro del contexto de la epidemia, y no son solo intentos de reproducir líneas de intervención que han tenido su origen a raíz de otras temáticas (prevención del tabaquismo, del uso de drogas, sedentarismo, etc.). En ese sentido incorpora no solo los desarrollos académicos y técnicos sobre investigación social de la epidemia, sino todo el bagaje de la acción a nivel comunitario y político en torno a ella. Es de estos aprendizajes que surge el concepto de vulnerabilidad, como una forma de visibilizar aquellos condicionantes estructurales en los cuales los sujetos nos hallamos insertos, y los cuales nos impiden adoptar conductas "no riesgosas." Las intervenciones estructurales son el mejor ejemplo de cómo este concepto fue incorporado a la prevención. Sin embargo, de manera paralela se fue abriendo paso el concepto de riesgo como construcción social al interior 
de esas prácticas, un ejemplo podría ser la estrategia de movilización comunitaria, pero por sobre todo, aquella que se funda en la Teoría de la Transformación social y el empoderamiento colectivo. Para algunos autores, como Parker (2000) este concepto viene a oponerse al concepto de riesgo tal como ha sido concebido tradicionalmente. Sin embargo, creemos que es posible establecer un diálogo entre la vulnerabilidad como concepto y una noción riesgo sexual entendido, no como un dato objetivo o dependiente solo de lo subjetivo, sino como una construcción social.

El concepto de vulnerabilidad surge en el campo de la epidemiología del VIH/SIDA como una tentativa de explicar cómo un modo específico de interrelación entre factores de diversa naturaleza -individuales, sociales y políticos- facilita o dificulta la exposición de una persona o de una población al VIH/SIDA (Villela 1998). En términos genéricos la vulnerabilidad es la relativa desprotección en la cual se puede encontrar un grupo de personas frente a potenciales daños de salud o amenaza a la satisfacción de sus necesidades básicas y al respeto de sus derechos humanos, en razón de determinadas características estructurales (biológicas, económicas, sociales, legales, culturales, etc.) (Cáceres 1998). De este modo, entenderemos vulnerabilidad como la relación entre la posibilidad de control real del riesgo que puede tener una persona o grupo de personas y su caracterización individual, sociocultural, económica y política, por lo que varía según las capacidades y recursos de cada persona y grupo.

Respecto del VIH/SIDA, la vulnerabilidad está condicionada por la capacidad o incapacidad del individuo o comunidad para controlar su riesgo de infección. Del mismo modo, remite a la forma que asume la relación entre el sujeto y el contexto en el cual se desenvuelve, condicionándole su competencia para prevenir. Estas interacciones dicen relación, básicamente, con la familia, con los servicios de salud, con la calidad de vida, las relaciones de poder (como las de género) y con la cultura respecto a la sexualidad. Estos aspectos contribuyen a colocar al sujeto en una situación de vulnerabilidad, ya sea por imitación de modelos o presiones socio-culturales que le incitan al riesgo y a la no prevención.

Siguiendo a Cáceres (1998) sostenemos que la vulnerabilidad frente al VIH/SIDA equivale a aquella fracción de los factores 
de riesgo para infección por VIH/SIDA y para progresión de la enfermedad, que es casi inmodificable por iniciativa del sujeto, en tanto tautológicamente deriva de la condición de vulnerabilidad social de una persona en tanto integrante de un grupo vulnerable. Es la fracción estructural del riesgo, aquella cuya reducción solo puede ser lograda por el sistema y finalmente es responsabilidad de éste.

A nuestro juicio, el mérito de la concepción de vulnerabilidad no es solo hacer visibles las condiciones de desventaja en que un sujeto se encuentra en relación a la epidemia, mostrando de alguna manera las redes y relaciones de poder en las cuales nos desenvolvemos los individuos, sino que también el hecho que permite redistribuir las responsabilidades en relación al riesgo. La culpa, la responsabilidad no es solo de quien se infecta, sino que se constituye una responsabilidad social en torno al tema, que interpela a todos los miembros de la sociedad, y en especial, a sus instituciones. Sin embargo, su utilización reproduce en cierta medida aquello que intenta criticar. Un énfasis tan marcado en lo estructural repone la dicotomía individuo-sociedad, y por otra parte, puede llevar al desvalimiento del actor social, en el sentido de que para el sujeto no existe la posibilidad real de cambiar su situación sino es a través de la modificación de estructuras altamente enraizadas, donde el esfuerzo individual e incluso colectivo parece una ingenuidad.

\section{Hacia un enfoque construccionista del riesgo sexual}

El enfoque construccionista del riesgo se debe en gran parte a los aportes de Mary Douglas desde la antropología cultural. Douglas defiende la noción de riesgo como un constructo social, la idea de que el riesgo es, a la postre, un proceso social. Su propósito es desmantelar, desde el análisis cultural, el enfoque de la percepción del riesgo, que sostiene que cada uno de nosotros es prisionero de sus esquemas cognitivos, y sostener la idea de que el conocimiento es un producto cambiante de la actividad social. Desde esta perspectiva, los riesgos no son concebidos ni como propiedades objetivas que dependen de cómo sea físicamente el mundo, ni como propiedades subjetivas que dependen de cómo 
sean cognitivamente los individuos. Los riesgos son construcciones sociales que dependen de factores socioculturales vinculados a estructuras sociales dadas. No se conciben como construcciones gratuitas, sino convencionales, en el sentido de que son entidades funcionales dentro de tales estructuras sociales: éstas permiten la distribución de la culpa y la responsabilidad, son utilizados como reclamo para la movilización social, permiten la adaptación de la conducta individual a pautas colectivas marcadas por la opinión pública, etc. (López y Lujan 2000). Este enfoque no presupone, a diferencia del enfoque cognitivo, una experiencia directa del individuo con respecto al riesgo; del mismo modo, en el tema de la "aceptabilidad del riesgo" (Douglas 1996) hace habitualmente del conflicto y los procesos sociales el centro de su investigación. La mayoría de los riesgos no pueden ser percibidos directamente por el individuo sino que estos les llegan a través de las instituciones (la ciencia, el gobierno, las ONG o los medios de comunicación). Por tanto, en la valoración de riesgos y aceptación de tecnologías (por ejemplo el uso del condón) no pesan demasiado los factores de carácter individual, sino que la aceptación depende más bien de aspectos tales como los valores sociales, la confianza en las instituciones o la transformación de la información en los medios (López y Lujan 2000). El enfoque es contextualizado, se centra en el estudio de los factores que hacen que determinados puntos de vista respecto al riesgo resulten dominantes en grupos sociales dados, o bien que se produzcan polarizaciones y enfrentamientos respecto de la distribución del riesgo. Destaca en este enfoque el hecho de que la noción de riesgo no tiene una base empírica o práctica, sino que esta construida culturalmente, lo que revela el hecho de que en determinados contextos sociales se destaquen ciertos riesgos, y se ignoren u oculten otros.

Para Mary Douglas la selectividad y las contradicciones toleradas, no son signos de debilidad perceptual, sino signos de una fuerte intención de proteger ciertos valores y las formas institucionales que los acompañan. Es decir, que el problema de determinar los niveles aceptables de riesgo, es una cuestión básicamente moral y política, ya que forma parte de determinar los niveles aceptables de vida y los niveles aceptables de moralidad y justicia social. Por ejemplo, los mensajes de prevención para el VIH/SIDA están orientados a la idea de pareja única, de la fidelidad en el matrimonio, etc. Lo que 
busca prevalecer es la institución de la familia y del matrimonio, a pesar de que la opción de pareja única para la prevención no puede adoptarse individualmente, silenciando el hecho de que la infidelidad masculina no es socialmente sancionada y que en algunos contextos puede incluso ser promovida. Siguiendo el mismo análisis, se puede entender que existen ciertas prácticas legítimas: el sexo heterosexual, por ejemplo, que es objeto de preocupación y atención, cuando este puede a conducir a un embarazo no planificado. Sin embargo, la misma práctica se deslegitima cuando ésta no tiene relación directa con la reproducción, sino que está más ligada al erotismo, y aunque puede exponer a la adquisición del VIH/SIDA o de otras ETS, no son objeto de la misma atención por parte de las autoridades. Por último, el abandono progresivo de la focalización de conductas de riesgo individuales, y la emergencia de una preocupación por los fenómenos interaccionales y socioculturales han permitido hacer sentido con la idea de que la sexualidad no se encuentra enraizada en el exclusivo dominio de lo subjetivo, sino que los significados y prácticas sexuales se encuentran socialmente organizados, es decir, con la noción de sexualidad como construcción social, que ha desarrollado un importante volumen de investigación a partir de los aportes de Foucault a la historia de la sexualidad, y a desarrollos más recientes de teóricos como Jeffrey Weeks y Gayle Rubín.

Una estrategia pensada desde puede este enfoque puede abrir paso a transformaciones más amplias que considere, pero a la vez cuestione, los valores que una sociedad desea para sus miembros, que incluya en su diseño no solo los contextos culturales y sociales, sino que también las historias personales que se ponen en juego en la adopción individual de estrategias preventivas. Que tenga la capacidad de movilizar a los sujetos como colectivos de acción, considerándolos no solo como objetos de intervención sino como actores sociales.

\section{A modo de conclusión}

La epidemia del VIH/SIDA ha tenido el valor de haberse configurado en un espacio social crítico que interpela al conjunto 
de la sociedad. El VIH/SIDA remece implacablemente los lugares más recónditos de la privacidad, enfrentando a cada persona con su sexualidad, su relación con el otro, con la intimidad de su individualidad. Cuando citamos a Jonathan Mann en relación a las fisuras de la sociedad por las cuales el VIH/SIDA ha hecho su recorrido en estas dos décadas desde su aparición, tenemos la sensación de visualizar una suerte de mapa, de radiografía de las tensiones que articulan nuestra sociedad. No parece casual que el VIH/SIDA afecte principalmente a los más pobres, a las mujeres, a los niños, a aquellas comunidades marginales dentro de las urbes modernas, a los homosexuales. La comunidad homosexual ha sido capaz de articularse y reaccionar de manera cohesionada ante la epidemia, de la cual fueron sus primeras víctimas. Si bien en un principio llevaron todo el peso de lo que significaba el virus, han logrado a través de su movilización visibilizarse como comunidad, avances importantes en relación a la reducción del contagio entre sus miembros. Sin embargo, todavía quedan las mujeres, como uno de los grupos más vulnerables, aumentando progresivamente las cifras de mujeres que viven con la enfermedad. Creemos que gran parte de la responsabilidad en este hecho se debe a la manera en como ha sido enfrentada la prevención hasta ahora. No es una negligencia casual el que se haya dado de esa manera. Sin duda, las relaciones de poder que mantienen a las mujeres en un lugar de subordinación no se tejen solo en la intimidad de la familia o en las relaciones laborales. El que los mensajes orientados a la prevención tengan una perspectiva eminentemente masculina, el que las intervenciones en sí mismas velen las tensiones existentes en los contextos sociales, que no hayan podido hasta ahora dar cuenta de la diversidad de maneras en que se organizan y significan las sexualidades, no puede ser atendido como un ocultamiento ni como una negligencia.

Asumimos que la incorporación de un concepto de riesgo entendido como una construcción social, permite dar cuenta de la manera en que las instituciones sociales normalizan ciertas reglas del juego. En verdad, que la investigación y la intervención social orientada a la prevención, como todo saber, no queda exenta del juego de producir y reproducir las diferencias de género. Sin embargo, el develamiento de esos juegos 
de poder abren el paso para la posibilidad de reconstruirlos, resignificarlos de una manera que sea más justa para hombres y mujeres.

\section{Nota}

1 Psicologa de la Universidad de Chile, Magíster en Estudios de Género y Cultura en América Latina, Mención Humanidades, Universidad de Chile. Investigadora y docente en la Universidad de Humanismo Cristiano.

\section{Bibliografía}

Beck, Ulrich. La sociedad del riesgo: hacia una nueva modernidad. Barcelona: Paidós, 1998.

Beriain, Josetxo comp. Las consecuencias perversas de la modernidad. Modernidad, Contingencia y Riesgo. Barcelona: Anthropos, 1996.

Cáceres, Carlos. "Dimensiones sociales y relevantes para la prevención del VIH/SIDA en América Latina y el Caribe." El SIDA en América Latina y el Caribe: una visión multidisciplinaria. Ed. José A. Izazola. México D.F.: Fundación Mexicana para la Salud/AC/SIDALAC, 1999.

Douglas, Mary. La aceptabilidad del riesgo según las ciencias sociales. Barcelona: Paidós, 1996.

Kendall, Cari. "The Construction of Risk in AIDS control programs." Eds. Richard Parker y John Gagnon. Conceiving Sexuality. Approaches to Sex Research in a Postmodern World. Londres: Routledge, 1995.

López, José A.y José L. Lujan. Ciencia y Política del Riesgo. Madrid: Alian$\mathrm{za}, 2000$.

Parker, Richard y John Gagnon, eds. Conceiving Sexuality. Approaches to Sex Research in a Postmodern World. Londres: Routledge, 1995.

Weeks, Jeffrey. Making sexual history. Cambridge: Polity Press, 2000. 